\title{
YBX1 promotes tumor progression via the PI3K/AKT signaling pathway in laryngeal squamous cell carcinoma
}

\author{
Jing Zhao, Pu Zhang, Xin Wang \\ Department of Otolaryngology, The Third Hospital of Hebei Medical University, Shijiazhuang, China \\ Contributions: (I) Conception and design: J Zhao; (II) Administrative support: The Third Hospital of Hebei Medical University; (III) Provision of \\ study materials or patients: J Zhao; (IV) Collection and assembly of data: P Zhang; (V) Data analysis and interpretation: P Zhang; (VI) Manuscript \\ writing: All authors; (VII) Final approval of manuscript: All authors. \\ Correspondence to: Jing Zhao. Medical Master, The Third Hospital of Hebei Medical University, No. 139 Ziqiang Road, Qiaoxi District, Shijiazhuang \\ 050051, China. Email: zhaojing19900321@163.com.
}

\begin{abstract}
Background: Laryngeal squamous cell carcinoma (LSCC) is one of the most commonly seen malignancies of the head and neck, with increasing incidence and mortality. The Y-box-binding protein 1 (YBX1) is a type of oncoprotein which is related to the malignant phenotype of many cancers. It is reported that YBX1 could regulate tumorigenesis, recurrence, and metastasis in multiple cancers. However, little is known about its carcinogenic function and mechanism in LSCC.

Methods: Firstly, Through Oncomine StarBase, we found that the YBX1 mRNA level was increased in a variety of cancer tissues, including in the LSCC, compared with normal tissues. We silenced YBX1 in LSCC cells using short hairpin RNAs (shRNAs). Secondly, the biological function of YBX1 in LSCC cells was examined by the Cell Counting Kit-8 (CCK-8) assay, flow cytometry, the wound healing assay, and the transwell assay. Thirdly, the correlation between YBX1 and the PI3K/AKT pathway was verified by the western blot assay.
\end{abstract}

Results: Expression of YBX1 is higher in a variety of cancer tissues, especially in the head and neck cancers. After transfected with lentiviral vectors, the expression of YBX1 was significantly silenced. Functionally, low expression of YBX1 promoted LSCC cell apoptosis and inhibited LSCC cell proliferation, migration, and invasion. The transfection of sh-YBX1 resulted in an obvious decrease in PI3K/AKT signaling molecules in LSCC cells.

Conclusions: We demonstrated that YBX1 could promote LSCC cell progression through the PI3K/AKT pathway, providing new insights into a potential biomarker and target for LSCC treatment.

Keywords: Laryngeal squamous cell carcinoma (LSCC); YBX1; PI3K/AKT; cancer promoter

Submitted Sep 09, 2021. Accepted for publication Nov 03, 2021.

doi: $10.21037 /$ tcr-21-2087

View this article at: https://dx.doi.org/10.21037/tcr-21-2087

\section{Introduction}

Laryngeal squamous cell carcinoma (LSCC) is one of the most common subtypes of laryngeal carcinoma (1). As one of the most common tumors of the head and neck, its onset is occult so that the proportion of patients initially diagnosed at an advanced stage (stage III or IV) is approximately $60 \%$ (2). LSCC mainly affects the population aged 40-60 years old, with a male-female ratio of 7-9:1 (3).
The important physiological functions of LSCC patients, such as breathing, vocalization, and swallowing, are severely impaired, which reduces their quality of life. Despite the great improvements in the treatment methods, such as radiotherapy, chemotherapy, and surgery, the 5 -year overall survival (OS) rate of LSCC has not significantly improved in the past 20 years (4). Therefore, there is an urgent need to reveal the pathogenesis of LSCC, determine its diagnostic biomarkers, and explore effective new therapeutic targets. 
As a member of the cold-shock protein superfamily, the Y-box-binding protein 1 (YBX1) is a nucleic acid binding protein, which is known to bind both DNA and RNA to affect transcriptional activation, DNA repair, and replication, as well as RNA processing and stability and protein translation (5). It is a well-established oncogenic transcriptional factor, and regulates tumorigenesis, recurrence, and metastasis in multiple cancers, such as gastric cancer, lung cancer, breast cancer, and pancreatic cancer (6-9). YBX1 directly binds to 3'-UTR of AURKA mRNA and promotes translation of AURKA mRNA, which can enhance the ability of proliferation and invasion of nasopharyngeal carcinoma cells (10). YBX1 also could sustain the rapid proliferation of bladder cancer cell through elevating glycolysis by targeting c-Myc and HIF1 $\alpha$, which act as the crucial transcription factors in glycolysis (11). In pancreatic ductal adenocarcinoma cells, GSK3B protein level is increased inducing cell-cycle arrest and cell proliferation because of the YBX1 binding to the GSK3B promoter near the transcription start site. Recent studies have shown that YBX1 is associated with head and neck cancer (12). Nevertheless, the relationship and molecular mechanism between YBX1 and LSCC has not been elucidated. In 2018, Zhao et al. found that YBX1 promotes LCSS progression (13). However, in that article, the cell functional experiments were only performed in the Hep-2 cell line. According to the Korean Cell Line Bank (https://cellbank.snu.ac.kr/main/index.html), the Hep2 cell line has been proved that it is positive for keratin by immunoperoxidase staining and the origin cells have been contaminated with Hela cells, which means that the results of the cell experiments are unreliable. In this study, in order to obtain more complete and reliable results, we explored the function of YBX1 and its potential underlying mechanism by using two different subtypes of LSCC cell lines (SNU1066, subtype: Glottic LSCC; SNU1076, subtype: Subglottic LSCC). We present the following article in accordance with the MDAR reporting checklist (available at https://dx.doi.org/10.21037/tcr-21-2087).

\section{Methods}

\section{Cell culture}

The SNU1066 and SNU1076 human LSCC cell lines were obtained from The First Affiliated Hospital of Sun Yatsen University which were procured from American Type Culture Collection (ATCC, Rockville, USA). Cells were grown in Dulbecco's modified Eagle's medium (DMEM) with RPMI-1640 containing 10\% fetal bovine serum (FBS), and were incubated at $5 \% \mathrm{CO}_{2}$ and $37^{\circ} \mathrm{C}$.

\section{$R N A$ extraction and quantitative real-time polymerase chain reaction ( $q R T-P C R)$}

According to the manufacturer's recommendation, we used the TRIzol reagent (Ambion, USA) to isolate the total RNA from LSCC cells, and reverse transcription was conducted with the FastQuant RT Kit (TIANGEN, China). GAPDH was used as the housekeeping gene for normalization and each sample was run in triplicate. Alterations in gene expression were expressed as fold change using the $2^{-\triangle \Delta C T}$ method. The PCR primer sequences were YBX1 forward: 5'-CCTAGAGAGGACGGCAATGA-3'; reverse: 5'-CGTCTGCGTCGGTAATTGAA-3'; GAPDH forward: 5'-GGGAAACTGTGGCGTGAT-3'; reverse: 5'-GAGTGGGTGTCGCTGTTGA-3'.

\section{Transfection assays and generation of stable clones}

Cells were transfected with lentiviral vectors to generate stable clones with low level YBX1 expression. The short hairpin RNA (shRNA) vectors for knockdown YBX1 expression (pLKO.1-CopGFP-YBX1-homo-416, the sequence AGCAGACCGTAACCATTATAG; pLKO.1-CopGFP-YBX1-homo-393, the sequence CCAGTTCAAGGCAGTAAATAT; pLKO.1-CopGFPYBX1-homo-771, the sequence GACGGCAATGAA GAAGATAAA) and the empty vector (pLKO.1-CopGFPshNC, the negative control, sh-NC) both contained a selection marker GFP. The 3 shRNAs were named shRNA1, sh-RNA2, and sh-RNA3 in turn. Following the instructions of the manufacturer, Lipofectamine 2000 (Invitrogen, USA) was used for cell transfection. After transfection, the expression efficiency was evaluated at $24 \mathrm{~h}$.

\section{Proliferation assay}

LSCC cells $\left(1 \times 10^{4} / \mathrm{mL}\right)$ transfected with PBS, sh-NC, or sh-YBX1 were inoculated into 96 -well plates. The wells were added with $10 \mu \mathrm{L}$ CCK-8 solution separately after incubation for different times $(24,48,72 \mathrm{~h})$. Cells were then incubated for another $4 \mathrm{~h}$ at $37^{\circ} \mathrm{C}$. The auto-microplate reader was used to measure the absorbance at $450 \mathrm{~nm}$ in each well. 


\section{Flow cytometric analysis}

LSCC cells $\left(1 \times 10^{6} / \mathrm{mL}\right)$ were treated with PBS, sh-NC, or sh-YBX1 for $48 \mathrm{~h}$, and were then harvested in different tubes. The Annexin V-FITC Apoptosis Detection Kit was used to measure apoptosis (Nanjing Keygen Biotech). The apoptosis level was determined by the flow cytometer (BD Biosciences, USA).

\section{Wound healing assay}

The SNU1066 and SNU1076 cells were treated with PBS, sh-NC, or sh-YBX1 when cells grew close to complete confluence. We used a $100-\mu \mathrm{L}$ pipette tip to scrape the cell plates in order to create the sterile wound gaps. An inverted microscope was used to photograph the spacing of the gaps at 0,24 , and $48 \mathrm{~h}$.

\section{Cell invasion assay}

After $24 \mathrm{~h}$ of transfection, the upper chambers of a Transwell apparatus with Matrigel (BD Biosciences) were seeded with cells $\left(1 \times 10^{5}\right)$ in $100 \mu \mathrm{L}$ RPMI-1640 medium. Medium containing $15 \%$ FBS was used to fill the lower chambers. We removed the cells on the upper surface of the membrane and fixed the cells which were attached to the lower surface of the membrane after $24 \mathrm{~h}$ of incubation at $37^{\circ} \mathrm{C}$. The fixed cells were stained and then counted in 4 randomly chosen regions.

\section{Western blot analysis}

Western blot assay was carried out using anti-PI3K (Rabbit, CST, 4249T), anti-p-AKT (Rabbit, CST, 4060), and anti-AKT (Rabbit, CST, 4691) antibodies, and goat anti-Rabbit IgG (Southern Biotech, 4050-05) conjugated to horseradish peroxidase (HRP) was used as the secondary antibody.

\section{Statistical analysis}

The SPSS 22.0 software (IBM, USA) was used to perform the statistical analysis. We reported the data as the mean \pm standard deviation of three independent experiments or more. For comparisons of multiple groups, Student's $t$-test (two tailed) or one-way analysis of variance (ANOVA) was used. Statistical significance was defined as differences with $\mathrm{P}<0.05$.

\section{Results}

\section{YBX1 expression is up-regulated in many cancers}

Through Oncomine StarBase (https://www.oncomine. org), we found that the $Y B X 1 \mathrm{mRNA}$ level was increased in a variety of cancer tissues compared with normal tissues (Figure 1). In addition, large sets of data showed that YBX1 mRNA levels were significantly increased in head and neck tumors compared with normal tissues (Figure 2). To investigate the function of YBX1 in LSCC, we silenced $Y B X 1$ expression in LSCC cells using 3 shRNAs, which were designated as sh-RNA1, sh-RNA2, and sh-RNA3. The sh-RNA3 suppressed the level of $Y B X 1$ mRNA expression with maximum efficiency compared with sh-RNA1 and sh-RNA2 (Figure 3A,3B). We chose sh-RNA3 to perform follow-up functional experiments and named it sh-YBX1.

\section{Inbibition of YBX1 diminishes proliferation and accelerates the apoptosis of LSCC cells}

To explore whether YBX1 expression correlates with LSCC progression, we conducted the CCK- 8 assay and the apoptosis assay. Compared with wild type and sh-NC, cell growth was significantly inhibited by the transfection of sh-YBX1 under the same conditions (Figure 3C,3D). YBX1 silencing caused a significant increase in the percentage of apoptotic cells in both cell lines (Figure 4). The results therefore demonstrated the pro-proliferative and antiapoptotic role of YBX1 in LSCC cells.

\section{Inbibition of YBX1 curbs the migration and invasion of LSCC cells}

Through the wound healing assay, the effect of $Y B X 1$ silencing on migration was evaluated in LSCC cells. The LSCC cells transfected with sh-YBX1 migrated much more slowly than the control groups, including wild type cells and sh-NC transfected cells at 24 and $48 \mathrm{~h}$ (Figure 5). Using the transwell assay, it was observed that LSCC cells with $Y B X 1$ knockdown showed decreased migration than the corresponding control groups (Figure 6A-6D). These results suggest that YBX1 contributes to the migratory and invasive capacity of LSCC cells.

\section{YBX1 exerts its function via the PI3K/AKT patbway}

It has been widely reported that the PI3K/AKT signaling pathway plays a major role in cancerous cytophysiology, 

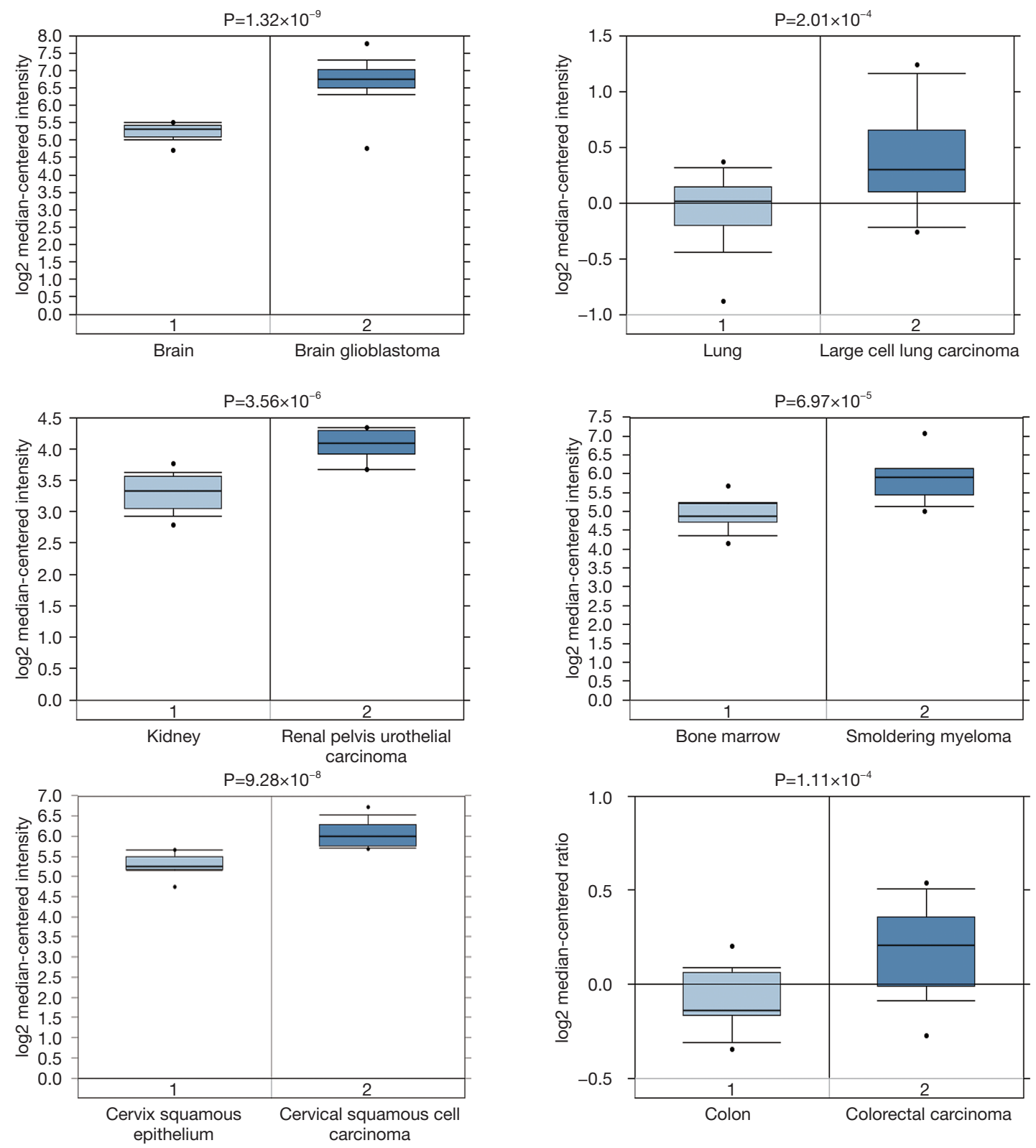

Figure 1 Box and whisker plots of Oncomine data on YBX1 mRNA levels (expressed as the $\log 2$ median-centered ratio) in various normal and cancerous tissues. $\mathrm{P}$ values, Student's $t$-test.

such as regulating the tumor cell cycle, survival, and motility. As shown in Figure 6E, low expression of YBX1 decreased the level of PI3K and p-AKT protein expression in LSCC cells. The results suggest that YBX1 may be an important player in the PI3K/AKT pathway.

\section{Discussion}

YBX1 was first recognized as a transcription factor. It was originally reported that $\mathrm{YBX} 1$ could specifically bind to the inverted CCAAT box sequences (5'-CTGATTGG-3'), called Y-boxes, which are in the promoter region of the major histocompatibility complex (MHC) class II genes (14). Nowadays, many studies have shown that YBX1 not only acts as a transcription factor but also as a translation regulator. YBX1, a 43-kDa protein containing 324 amino acids, represents the predominant form of the cold-shock protein (CSP) family (15). It consists of three parts: an 

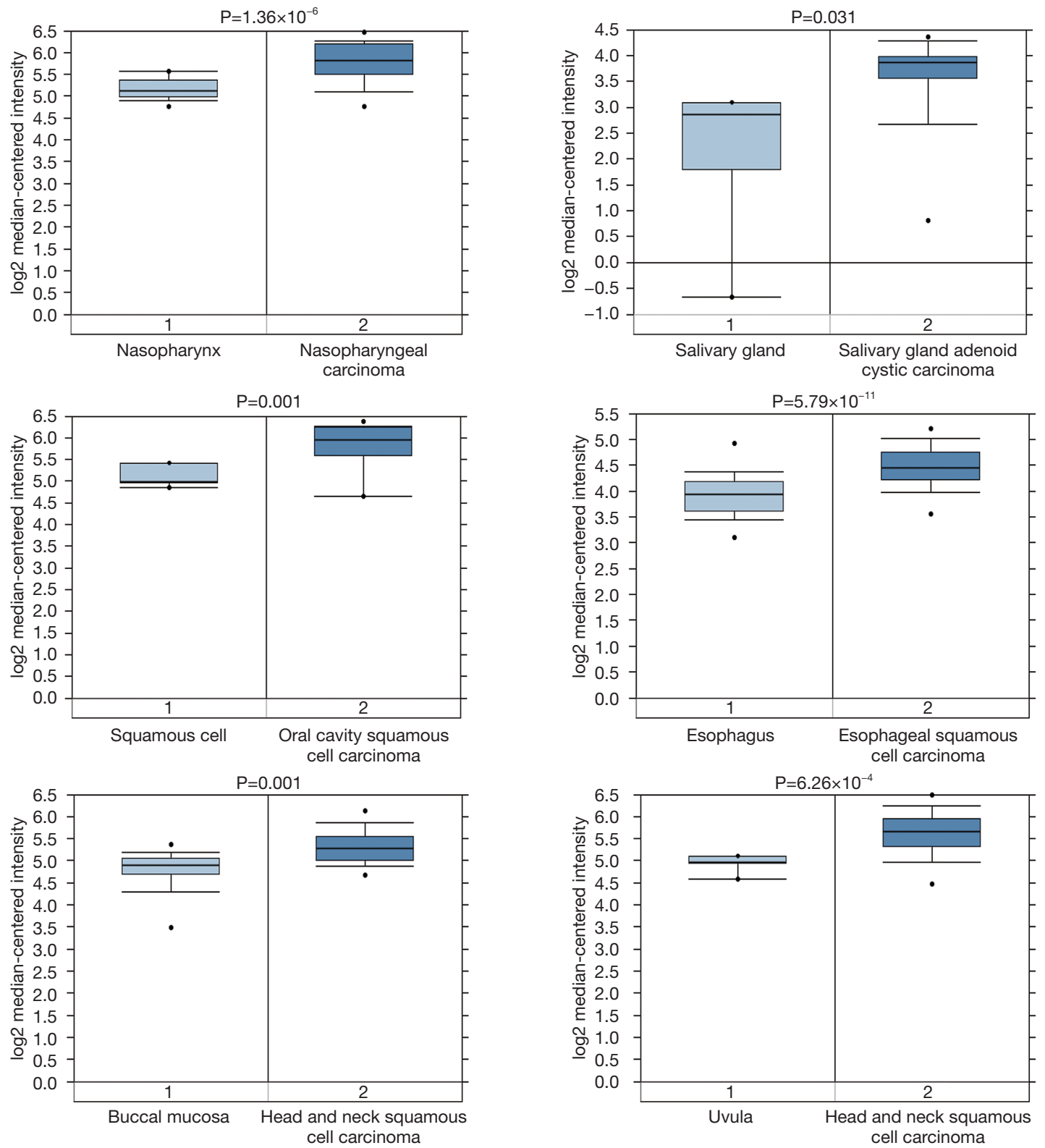

Figure 2 Box and whisker plots of Oncomine data on YBX1 mRNA levels (expressed as the $\log 2$ median-centered ratio) in normal and head and neck cancer tissues. $\mathrm{P}$ values, Student's $t$-test.

alanine/proline-rich $\mathrm{N}$-terminal domain (A/P domain), a highly conserved cold-shock domain (CSD), and a large C-terminal domain (CTD) $(16,17)$. The CTD is carried with alternating clusters of positively and negatively charged amino acid residues, and is involved in protein/ protein interaction and DNA/RNA binding. The CSD has nucleic acid binding properties so that it can function pleiotropically in various processes (18), for example cell proliferation, cell differentiation, and cell fate transition, among others.

By identifying YBX1 as a vital marker of tumor aggressiveness and malignant cell transformation and also as a promising molecular target in the therapy of inflammation and cancer, extensive research of YBX1 was further stimulated. Although the relationship between YBX1 and cancer cell progression has been established in many 

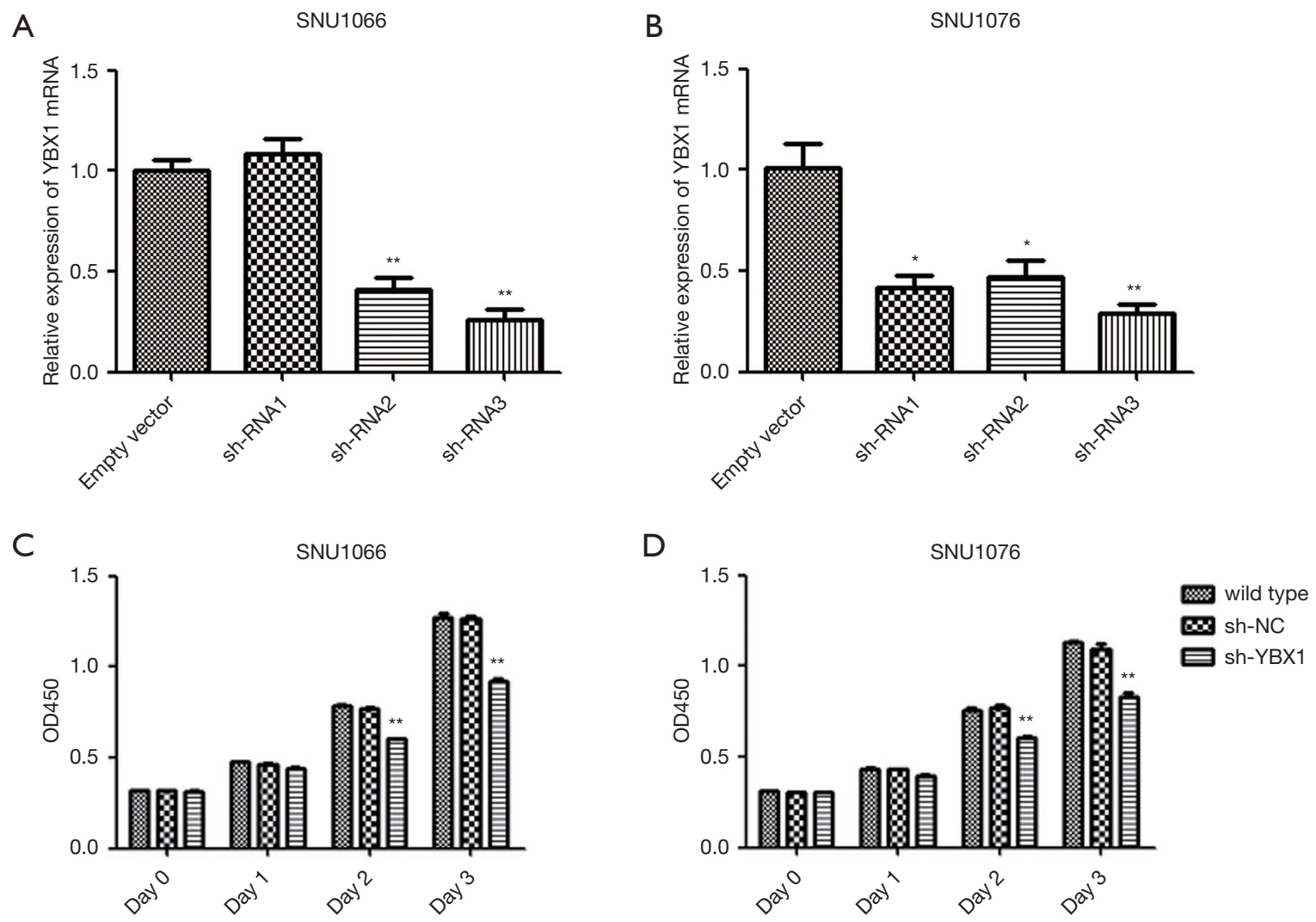

Figure 3 The expression of $Y B X 1$ could been significantly silenced after transfection of sh-RNA and inhibition of YBX1 diminishes proliferation of LSCC cells. (A,B) SNU1066 and SNU1076 cells were transfected with empty vector, sh-RNA1, sh-RNA2 or sh-RNA3 to knock down $Y B X 1$ gene expression. The expression levels of $Y B X 1 \mathrm{mRNA}$ was measured by qRT-RCR. The expression levels of $Y B X 1$ mRNA were significantly lower in the $Y B X 1$ sh-RNA2 or sh-RNA3 transfected groups compared with the control group. (C,D) CCK-8 assay was conducted for cell proliferation analysis. Cell proliferation was significantly lower in the sh-YBX1 transfected groups compared with the control groups. * $\mathrm{P}<0.05$; **, $\mathrm{P}<0.01$. LSCC, laryngeal squamous cell carcinoma; CCK-8, Cell Counting Kit-8.

malignant tumors, the effect of YBX1 on LSCC has been rarely studied. In order to better understand the functional role of YBX1 in LSCC, we knocked down YBX1 expression in two highly aggressive LSCC cell lines, namely SNU1066 and SNU1076. Down-regulated expression of YBX1 caused a decrease in proliferation, migration, and invasive potential and an increase in the apoptosis of LSCC cells. The results indicate that YBX1 functions as a carcinogen in LSCC.

It has already been reported that YBX1 can act as a signal transducer for multiple growth factors (19-23), such as EGFR, NOTCH, WNT, PDGF, and HER3, suggesting that YBX1 plays a key role in regulating malignant cell proliferation. Additionally, it was found that inhibition of YBX1 was accompanied by down-regulation of Cyclin D1, P16, $\mathrm{P} 21$, and Bcl-2 $(24,25)$ and up-regulation of pro-apoptotic regulators including APAF-1, BAX, and NOXA (26), which means that YBX1 also plays an important role in cellular apoptosis. Through controlling cell cyclerelated genes, YBX1 regulates not only the G1 phase but also the $G 2 / M$ phases $(27,28)$. Furthermore, it is widely acknowledged that YBX1 is a potent inducer of epithelialmesenchymal transition, and is also a strong contributor to the metastatic cascade (29), which means that YBX1 has a significant effect on cell metastasis. Many studies indicate that up-regulation of YBX1 can promote the creation of blood vessels in tumors and provide the route for metastatic dissemination of malignant cells (30). Moreover, it has been shown that YBX1 has the ability to induce the formation of more spindle-shaped cells, meaning that YBX1 can increase the invasiveness of tumors. More and more studies have proven that YBX1 can promote the development of many cancers. 

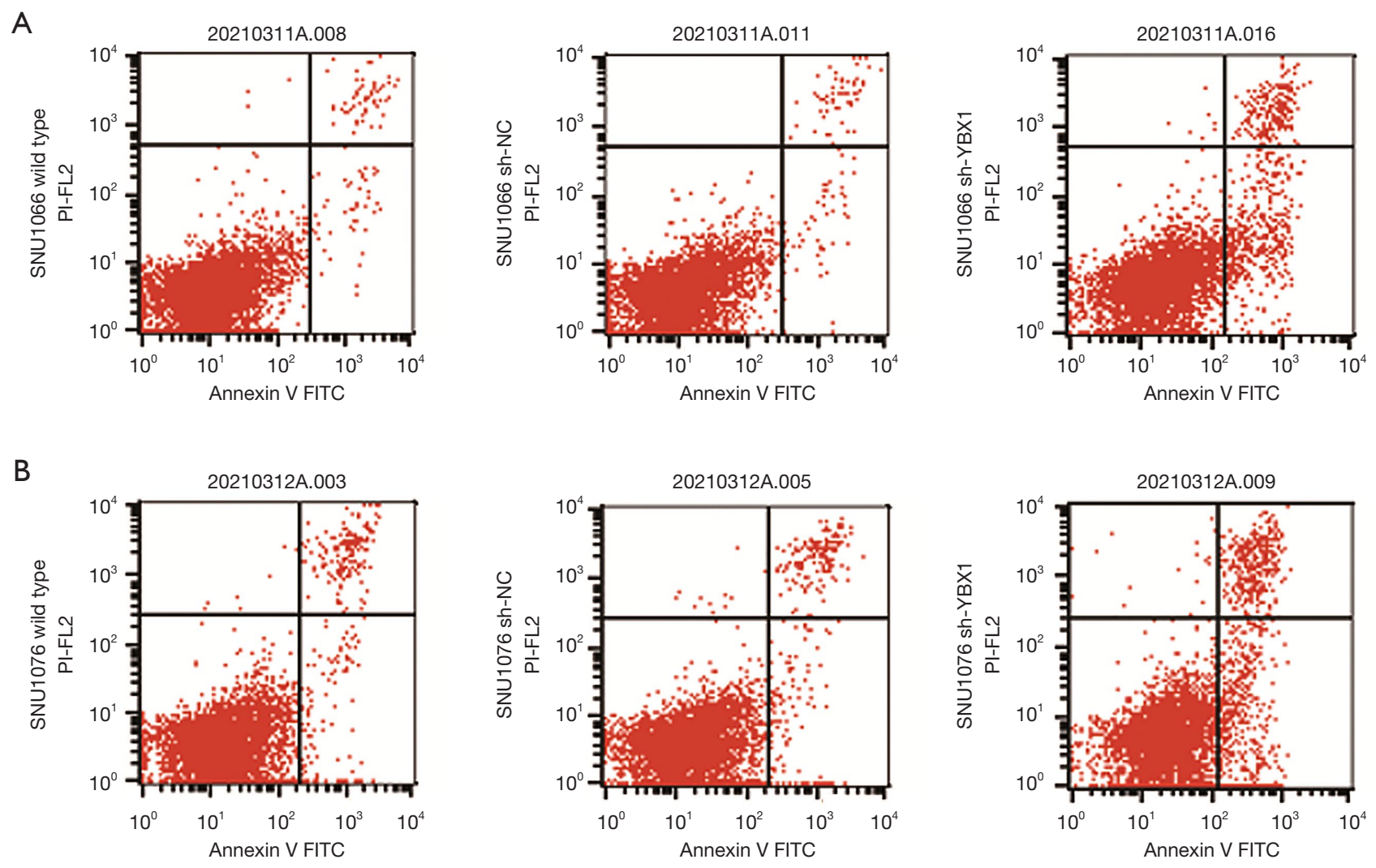

C

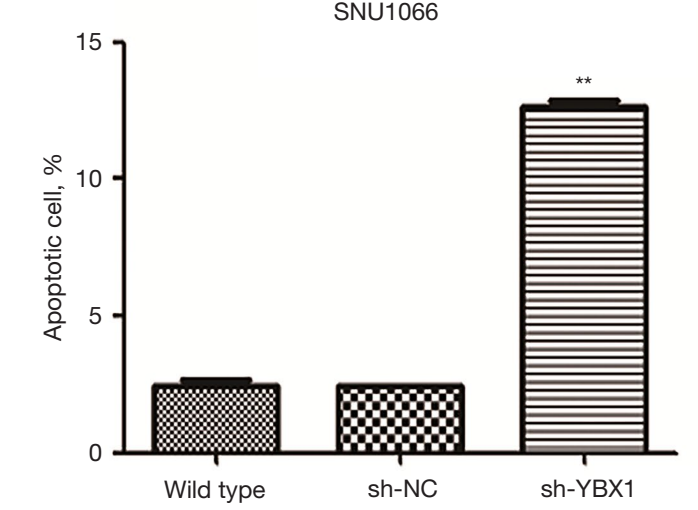

D

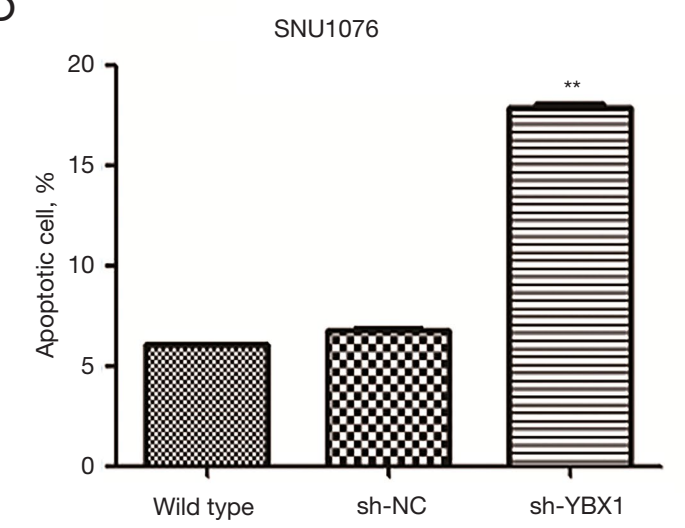

Figure 4 Inhibition of YBX1 accelerates apoptosis of LSCC cells. At $48 \mathrm{~h}$ after transfection, cell apoptosis was analyzed by flow cytometry. Late apoptotic cells increased significantly after $48 \mathrm{~h}$ transfection with sh-YBX1 compared to the control groups in SNU1066 (A,C) and SNU1076 (B,D) cells. **, $\mathrm{P}<0.01$. LSCC, laryngeal squamous cell carcinoma.

The PI3K/AKT signaling pathway plays a key role in cancerous cytophysiology in human cancers, such as cutaneous squamous cell carcinoma (31), brain cancer (32), prostate cancer (33), and ovarian cancer (34). It has been demonstrated that YBX1 can activate the PI3K/AKT kinase signaling pathway in many cancers (35). To date, however, no direct connection between YBX1 and the PI3K/AKT pathway in LSCC has been reported. Through the western blot analysis, the results showed that YBX1 could regulate PI3K and p-AKT levels, demonstrating that knockdown 
A
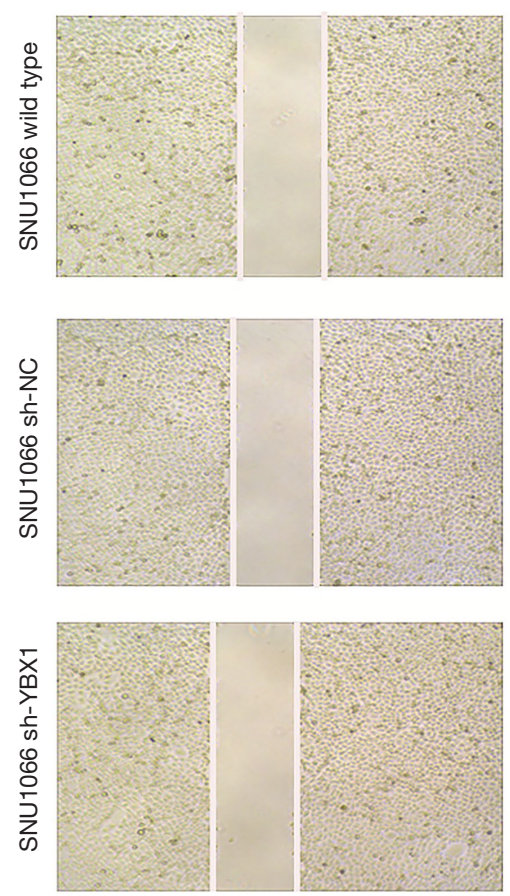

B
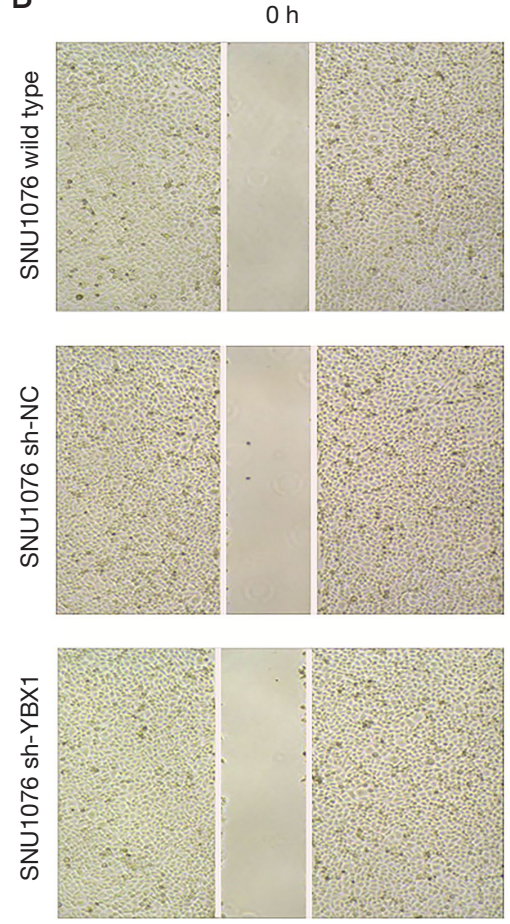

$24 \mathrm{~h}$
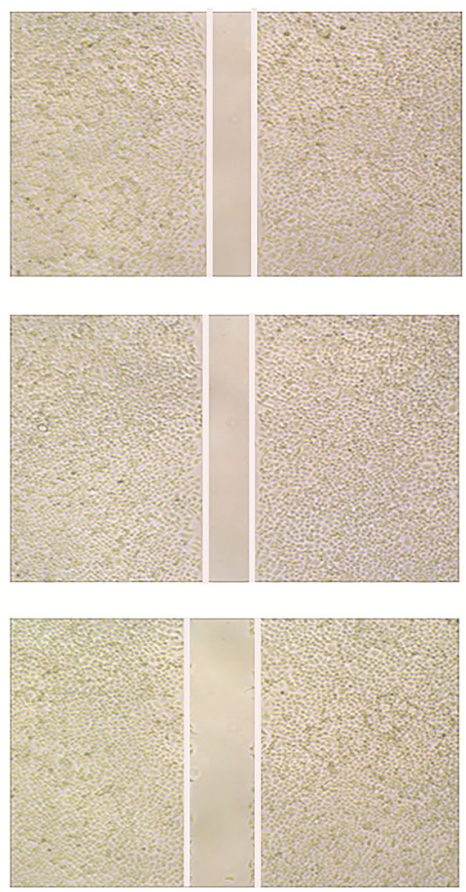

$24 \mathrm{~h}$
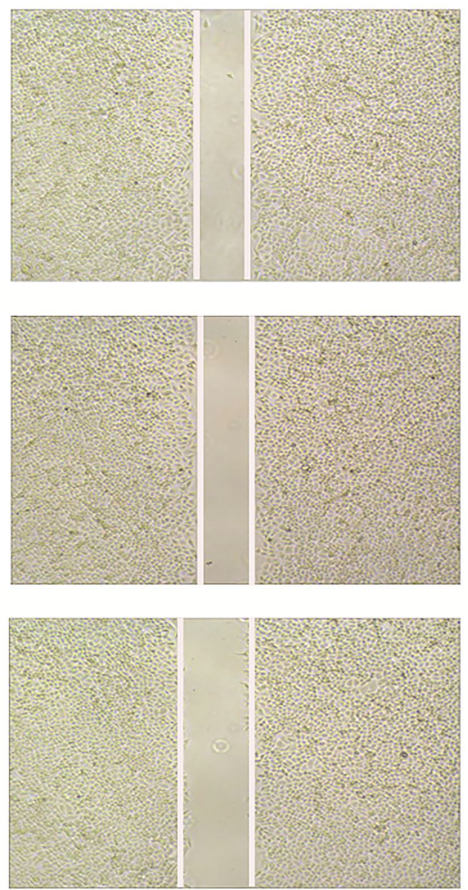

$48 \mathrm{~h}$
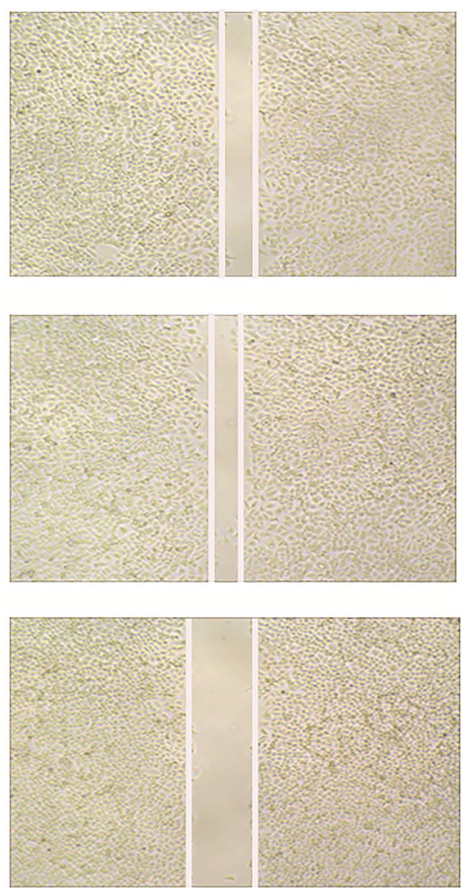

$48 \mathrm{~h}$
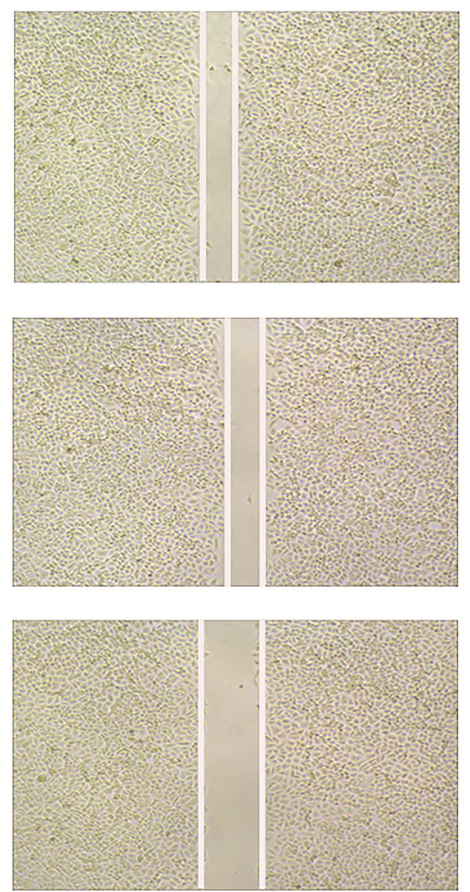

Figure 5 Inhibition of YBX1 curbs the migration of LSCC cells. Wound healing assay showed that SNU1066 (A) and SNU1076 (B) cell motility could be effectively suppressed by sh-YBX1 compared by the control groups (magnification: 40x). LSCC, laryngeal squamous cell carcinoma. 
A

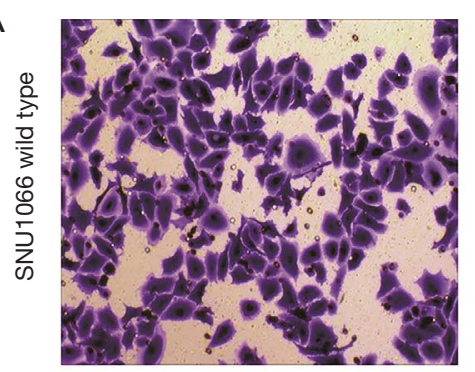

B

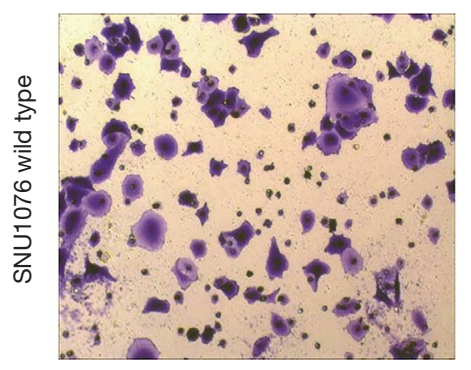

C

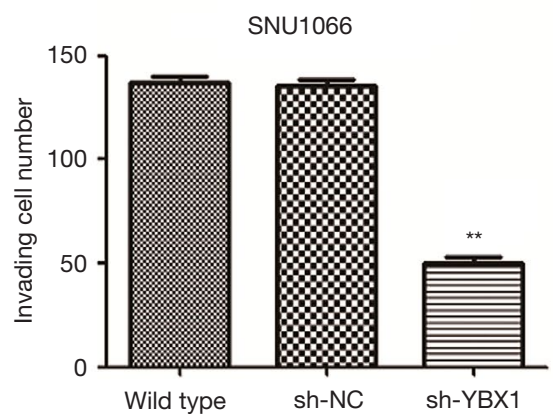

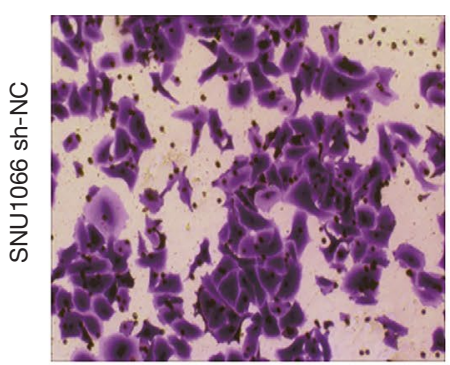
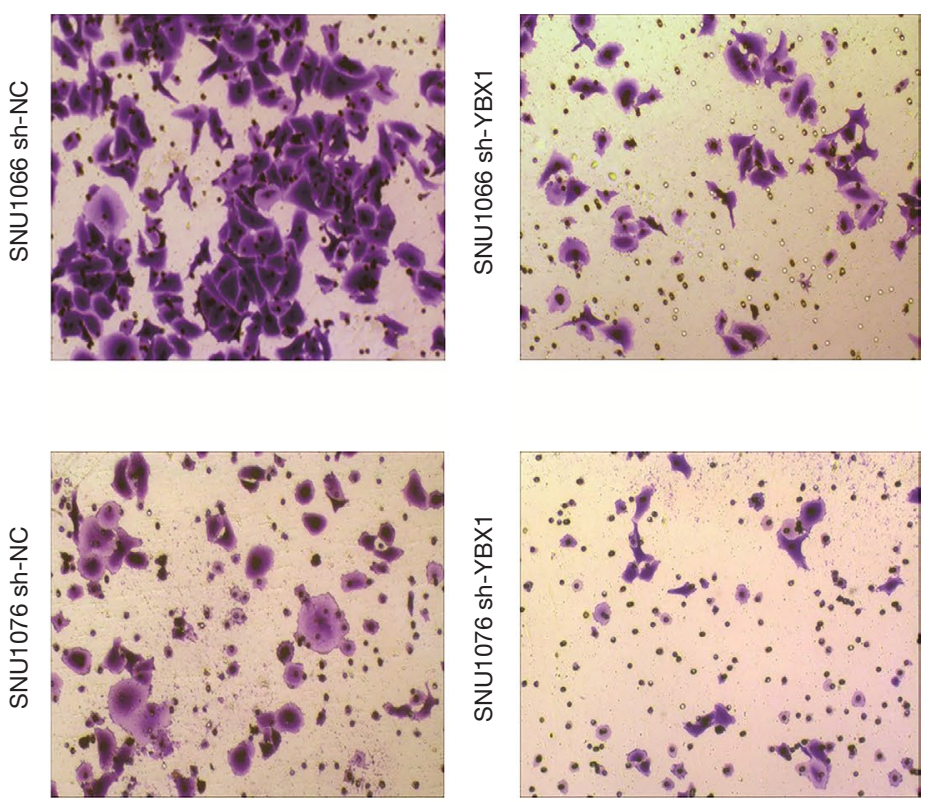

D

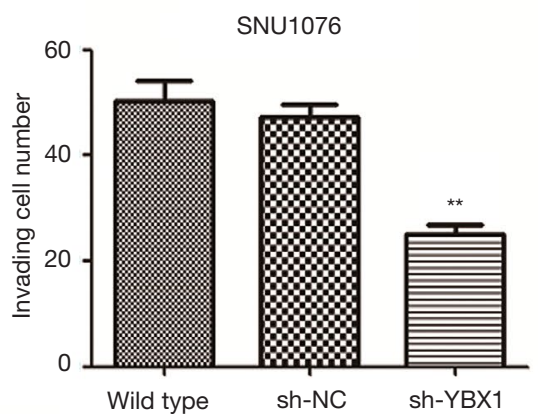

$E$

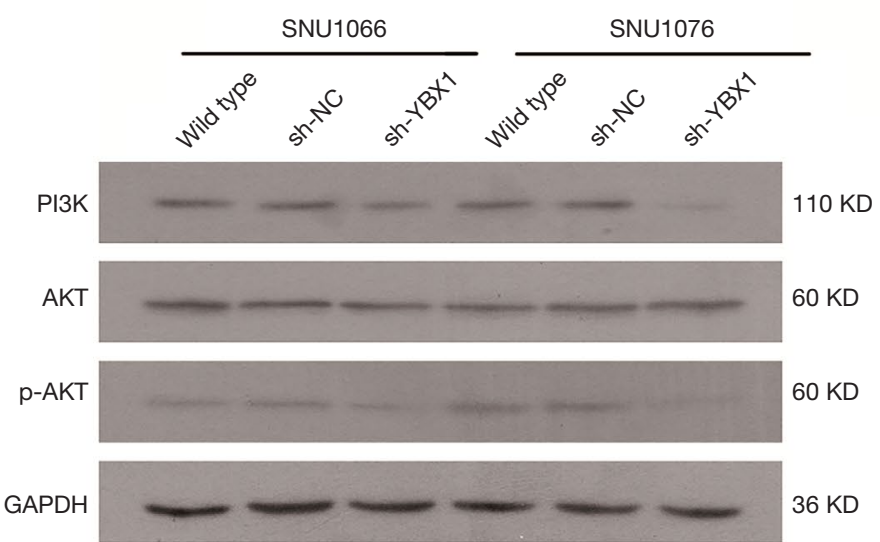

Figure 6 Inhibition of YBX1 curbs the invasion of LSCC cells and YBX1 exerts its function via the PI3K/AKT pathway. The cells that invaded through the membrane were fixed, stained with crystal violet. Downregulation of YBX1 in SNU1066 (A,C) and SNU1076 (B,D) significantly inhibited invasion compared with the control groups (magnification: 100x). (E) Western bolt was used for analyzing the protein level of PI3K, AKT, p-AKT. PI3K and p-AKT decreased significantly in sh-YBX1 transfected groups compared with the control groups in SNU1066 and SNU1076 cells. **, $\mathrm{P}<0.01$. LSCC, laryngeal squamous cell carcinoma. 
of YBX1 led to decreased expression of critical factors of the PI3K/AKT pathway. YBX1 can positively control the expression of $\mathrm{PI} 3 \mathrm{~K}$ and $\mathrm{p}-\mathrm{AKT}$ proteins, which plays a major role in the development of LSCC.

In conclusion, our studies indicated that YBX1 functions as a carcinogen, and the inhibition of YBX1 showed negative effects in activating the PI3K/AKT signaling pathway in LSCC. The clinical application of this YBX1/PI3K/AKT axis needs further in vivo and clinical investigation. YBX1 has a close relationship with the progression and prognosis of LSCC, and it may hopefully serve as a novel target for LSCC therapy in the future.

\section{Acknowledgments}

Funding: The work was funded by grants from the Natural Science Foundation of Hebei Province of China (No. H2019206296).

\section{Footnote}

Reporting Checklist: The authors have completed the MDAR reporting checklist. Available at https://dx.doi. org/10.21037/tcr-21-2087

Conflicts of Interest: All authors have completed the ICMJE uniform disclosure form (available at https://dx.doi. org/10.21037/tcr-21-2087). The authors have no conflicts of interest to declare.

Ethical Statement: The authors are accountable for all aspects of the work in ensuring that questions related to the accuracy or integrity of any part of the work are appropriately investigated and resolved.

Open Access Statement: This is an Open Access article distributed in accordance with the Creative Commons Attribution-NonCommercial-NoDerivs 4.0 International License (CC BY-NC-ND 4.0), which permits the noncommercial replication and distribution of the article with the strict proviso that no changes or edits are made and the original work is properly cited (including links to both the formal publication through the relevant DOI and the license). See: https://creativecommons.org/licenses/by-nc-nd/4.0/.

\section{References}

1. Bray F, Ferlay J, Soerjomataram I, et al. Global cancer statistics 2018: GLOBOCAN estimates of incidence and mortality worldwide for 36 cancers in 185 countries. CA Cancer J Clin 2018;68:394-424.

2. Steuer CE, El-Deiry M, Parks JR, et al. An update on larynx cancer. CA Cancer J Clin 2017;67:31-50.

3. Bradley PJ. Laryngeal cancer in nondrinker nonsmoker young patients: a distinct pathological entity? Curr Opin Otolaryngol Head Neck Surg 2016;24:140-7.

4. Liu W, Zhang C, Gong X, et al. Prognostic value of immune-related genes in laryngeal squamous cell carcinoma. Transl Cancer Res 2020;9:6287-302.

5. Eliseeva IA, Kim ER, Guryanov SG, et al. Y-box-binding protein 1 (YB-1) and its functions. Biochemistry (Mosc) 2011;76:1402-33.

6. Lim JP, Shyamasundar S, Gunaratne J, et al. YBX1 gene silencing inhibits migratory and invasive potential via CORO1C in breast cancer in vitro. BMC Cancer 2017;17:201.

7. Cui Y, Li F, Xie Q, et al. YBX1 mediates autophagy by targeting $\mathrm{p} 110 \beta$ and decreasing the sensitivity to cisplatin in NSCLC. Cell Death Dis 2020;11:476.

8. Zhang E, He X, Zhang C, et al. A novel long noncoding RNA HOXC-AS3 mediates tumorigenesis of gastric cancer by binding to YBX1. Genome Biol 2018;19:154.

9. Liu Z, Li Y, Li X, et al. Overexpression of YBX1 Promotes Pancreatic Ductal Adenocarcinoma Growth via the GSK3B/Cyclin D1/Cyclin E1 Pathway. Mol Ther Oncolytics 2020;17:21-30.

10. Ban $Y$, Tan $Y$, Li X, et al. RNA-binding protein YBX1 promotes cell proliferation and invasiveness of nasopharyngeal carcinoma cells via binding to AURKA mRNA. J Cancer 2021;12:3315-24.

11. Xu L, Li H, Wu L, Huang S. YBX1 promotes tumor growth by elevating glycolysis in human bladder cancer. Oncotarget 2017;8:65946-56.

12. Zhou LL, Ni J, Feng WT, et al. High YBX1 expression indicates poor prognosis and promotes cell migration and invasion in nasopharyngeal carcinoma. Exp Cell Res 2017;361:126-34.

13. Zhao X, Zhang W, Ji W. YB-1 promotes laryngeal squamous cell carcinoma progression by inducing miR155 expression via c-Myb. Future Oncol 2018;14:1579-89.

14. Didier DK, Schiffenbauer J, Woulfe SL, et al. Characterization of the cDNA encoding a protein binding to the major histocompatibility complex class II Y box. Proc Natl Acad Sci U S A 1988;85:7322-6.

15. Lindquist JA, Mertens PR. Cold shock proteins: from cellular mechanisms to pathophysiology and disease. Cell 
Commun Signal 2018;16:63.

16. Lyabin DN, Eliseeva IA, Ovchinnikov LP. YB-1 protein: functions and regulation. Wiley Interdiscip Rev RNA 2014;5:95-110.

17. Kloks CP, Spronk CA, Lasonder E, et al. The solution structure and DNA-binding properties of the cold-shock domain of the human Y-box protein YB-1. J Mol Biol 2002;316:317-26.

18. Budkina KS, Zlobin NE, Kononova SV, et al. Cold Shock Domain Proteins: Structure and Interaction with Nucleic Acids. Biochemistry (Mosc) 2020;85:S1-S19.

19. Nagasu S, Sudo T, Kinugasa T, et al. Y-box-binding protein 1 inhibits apoptosis and upregulates EGFR in colon cancer. Oncol Rep 2019;41:2889-96.

20. Breitkopf DM, Jankowski V, Ohl K, et al. The YB1:Notch-3 axis modulates immune cell responses and organ damage in systemic lupus erythematosus. Kidney Int 2020;97:289-303.

21. Chao HM, Huang HX, Chang PH, et al. Y-box binding protein-1 promotes hepatocellular carcinoma-initiating cell progression and tumorigenesis via $\mathrm{Wnt} / \beta$-catenin pathway. Oncotarget 2017;8:2604-16.

22. van Roeyen CR, Eitner F, Martinkus S, et al. Y-box protein 1 mediates PDGF-B effects in mesangioproliferative glomerular disease. J Am Soc Nephrol 2005;16:2985-96.

23. Kashihara M, Azuma K, Kawahara A, et al. Nuclear Y-box binding protein-1, a predictive marker of prognosis, is correlated with expression of HER2/ErbB2 and HER3/ ErbB3 in non-small cell lung cancer. J Thorac Oncol 2009;4:1066-74.

24. Schittek B, Psenner K, Sauer B, et al. The increased expression of $\mathrm{Y}$ box-binding protein 1 in melanoma stimulates proliferation and tumor invasion, antagonizes apoptosis and enhances chemoresistance. Int J Cancer 2007;120:2110-8.

25. Fujii T, Seki N, Namoto-Matsubayashi R, et al. YB-1 prevents apoptosis via the mTOR/STAT3 pathway in HER-2-overexpressing breast cancer cells. Future Oncol
2009;5:153-6

26. Zhang YF, Homer C, Edwards SJ, et al. Nuclear localization of Y-box factor YB1 requires wild-type p53. Oncogene 2003;22:2782-94.

27. Khandelwal P, Padala MK, Cox J, et al. The N-terminal domain of $y$-box binding protein-1 induces cell cycle arrest in $\mathrm{g} 2 / \mathrm{m}$ phase by binding to cyclin $\mathrm{d} 1$. Int $\mathrm{J}$ Cell Biol 2009;2009:243532.

28. Kotake Y, Arikawa N, Tahara K, et al. Y-box Binding Protein 1 Is Involved in Regulating the G2/M Phase of the Cell Cycle. Anticancer Res 2017;37:1603-8.

29. Kosnopfel C, Sinnberg T, Sauer B, et al. YB-1 Expression and Phosphorylation Regulate Tumorigenicity and Invasiveness in Melanoma by Influencing EMT. Mol Cancer Res 2018;16:1149-60.

30. Takahashi M, Shimajiri S, Izumi H, et al. Y-box binding protein-1 is a novel molecular target for tumor vessels. Cancer Sci 2010;101:1367-73.

31. Janus JM, O'Shaughnessy RFL, Harwood CA, et al. Phosphoinositide 3-Kinase-Dependent Signalling Pathways in Cutaneous Squamous Cell Carcinomas. Cancers (Basel) 2017;9:86.

32. Mantamadiotis T. Towards Targeting PI3K-Dependent Regulation of Gene Expression in Brain Cancer. Cancers (Basel) 2017;9:60.

33. Crumbaker M, Khoja L, Joshua AM. AR Signaling and the PI3K Pathway in Prostate Cancer. Cancers (Basel) 2017;9:34.

34. Piddock RE, Bowles KM, Rushworth SA. The Role of PI3K Isoforms in Regulating Bone Marrow Microenvironment Signaling Focusing on Acute Myeloid Leukemia and Multiple Myeloma. Cancers (Basel) 2017;9:29.

35. Evdokimova V, Ruzanov P, Anglesio MS, et al. Aktmediated YB-1 phosphorylation activates translation of silent mRNA species. Mol Cell Biol 2006;26:277-92.

(English Language Editor: C. Betlzar)
Cite this article as: Zhao J, Zhang $\mathrm{P}$, Wang X. YBX1 promotes tumor progression via the PI3K/AKT signaling pathway in laryngeal squamous cell carcinoma. Transl Cancer Res 2021;10(11):4859-4869. doi: 10.21037/tcr-21-2087 